\title{
GPPS-BJ-2019-0219
}

\section{Experimental Research on 3D Particle Tracking Velocimetry Based on Light Field Imaging}

\author{
Huifang Liu \\ University of Shanghai for Science and \\ Technology \\ 1584765119@qq.com \\ Yangpu, Shanghai, China
}

\author{
Xiaoshu Cai \\ University of Shanghai for \\ Science and Technology \\ usst caixs@163.com \\ Yangpu, Shanghai, China
}

\author{
Tianlei Qian \\ University of Shanghai for \\ Science and Technology \\ chantylay@qq.com \\ Yangpu, Shanghai, China
}

\author{
Wu Zhou \\ University of Shanghai for Science and \\ Technology \\ usst_wzhou@163.com \\ Yangpu, Shanghai, China
}

\section{ABSTRACT}

A micro PTV (Particle Tracking Velocimetry) system is established based on a commercial light field camera (Raytrix R12) without knowing the inner optical parameters. The homemade image processing algorithms are established and used for processing of light filed images. The refocusing technique is used for depth estimation, making use of the relation between the optimal refocus coefficient $\alpha_{o p t}$ and the object depth $d$ in the measuring range. In order to recognize the particle images, all-in-focus image is obtained based on the principle of maximum sharpness. For the particle tracking, two different methods are compared, i.e., double-exposure double-frame PTV and single-exposure single-frame PTV. The system, including hardware and image processing program, is calibrated using a dot calibration target. The depth calibration curve is fitted by least squares method. The measuring range, depth resolution and the measuring error are analysed by experiments. The system was applied to a turbulent flow over a flat plate with two different exposure time. The above-mentioned two PTV methods are complementary to get both Euler and Lagrange information, using a light field camera with very low frame rate.

Key words: light field imaging; refocusing; depth estimation; 3D PTV

\section{INTRODUCTION}

The flow in nature can be seen everywhere, such as when the aircraft is flying, the wing is vibrated by the turbulence of the airflow, and the blood flow in the blood vessels of the human body. Accurate measurement of flow velocity is of great significance for aerospace and human cardiovascular disease prevention. However, the flow itself is three- dimensional, and the traditional 2D PTV technique for measuring the plane velocity field has been unable to meet the needs of the deeper research of most flow mechanisms. Therefore, it is urgent to measure the 3D velocity field in the flow, and then explore the nature of more interesting flow phenomena.

The current 3D PTV technology mainly includes stereoscopic PTV (SPTV) (Willneff $\mathbf{J}$ et al, 2002), digital defocus PTV (DDPTV)( Pereira F et al, 2006), scanning PTV (SSPTV)( Hoyer K et al, 2005), holographic PTV(HPTV)

(Satake S et al, 2006) et al. While these flow field measurement technologies either have complicated systems or have high costs, which limits its practical application.

In recent years, the advantages of light field imaging technology due to its single-camera single exposure to obtain 3D information have aroused the research enthusiasm of researchers in many fields. In the field of flow field measurement, there are light field PIV (particle image velocimetry) technology for obtaining Euler information and light field PTV (particle tracking speed measurement) technology for obtaining Lagrangian information. Fahringer(2015) and Shi S(2017) have made good progress in the measurement of PIV flow field in the light field. They use a flow field reconstruction method similar to tomography PIV, which divides the measured flow field into multiple voxels, then reconstructs the voxel intensity by ray tracing combined with MART method. At last the 3D velocity of the flow field is obtained by combining the $3 \mathrm{D}$ cross-correlation algorithm. When it is necessary to track the motion trajectory of a single particle, especially for the case where the flow field Lagrangian information needs to be obtained, the 3D PTV technology shows an advantage, and the research of the light 
field PTV is less than the light field PIV. Bajpayee (2013) studied the particle localization method of the high-speed light field camera to measure the flow field. They applied adaptively single-threshold segmentation to identify the particles on each refocus image. The maximum depth and minimum depth at which the refocus image can be detected by the same particle is averaged as the depth of the particle. Combined with the 3D PTV technology, proved the feasibility of measuring the flow field by the light field PTV. Ohmi (2016) extracted two different perspective images from the raw light field image and obtained their parallax. The Taylor's polynomial fitting method was used to fit the relationship between depth and parallax, then combined with threedimensional PTV technology to measure the flow field in a square sink (magnetic stirrer agitation). Although light field imaging combined with particle tracking technology has been applied to 3D flow field measurement, more detailed work is still needed in depth measurement methods and accuracy.

This paper mainly studies the light field 3D PTV technology and is structured as follows. In section 1, the theoretical background of depth measurement of light field camera and the principle of 3D PTV are introduced. This includes details about parameterization and refocusing of light field, the principle of depth estimation and main image processing process for single-exposure single-frame PTV et al. In section 2, the optical field imaging system is built. The experiments for calibration are performed and the error is analysed. In section 3 , the measurement experiment of flow field is applied to the flat plate turbulence test bench.

\section{PLENOPTIC PTV}

\subsection{Light field imaging}

\subsubsection{Parameterization and refocusing of the light field}

The light field is actual the total of all the light radiation functions in space. The light field can be parameterized by rays and two parallel planes, which are intersected with the rays (Levoy M et al, 1996). As shown in Figure 1 (a), Plane $U$ - $V$ is the plane where the microlens is located, and plane $X$ $Y$ is the plane where the optical detector is located. $l$ is the distance between two parallel planes. $L(u, v, x, y)$ represents the radiant intensity of the light beam passing through the point $(u, v)$ on the plane $U-V$ and the point $(x, y)$ on the $X-Y$ plane. The normal angle is $\theta$. Introducing a paraxial approximation and ignoring the change in distance between the two planes, the total energy received from the beam can be received by the following equation:

$$
I(x, y)=\iint L(u, v, x, y) d u d v
$$

The raw plenoptic image is a $2 \mathrm{D}$ image that records both the angular and spatial information in a scene. Refocusing is to extract the $4 \mathrm{D}$ light field from the raw 2D light field image, and thenre-project the obtained 4D light field to form a 2D image on the new imaging plane(Ng R et al, 2005). Figure 1(b) shows a simplified refocusing schematic of the 2D light field.

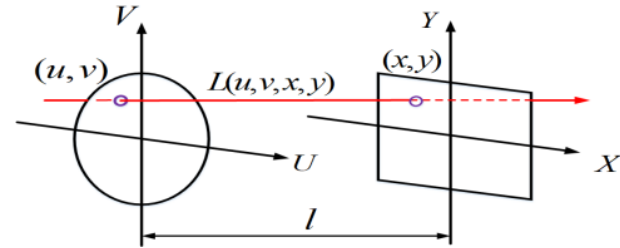

(a)

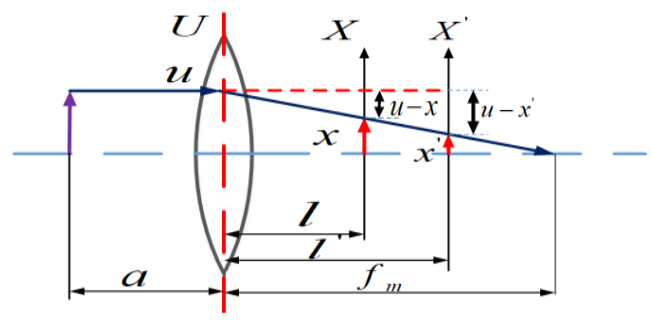

(b)

\section{Figure 1 (a) Light Field Biplane Parameterization Diagram;(b) Simplified Refocusing Schematic}

Where $u$ is the intersection coordinate of the beam $L$ and the plane $U$ of the microlens, $x$ is the intersection coordinate of the beam $L$ and the plane $X$ of the detector, and $x$ ' is the intersection coordinate of the beam $L$ and the refocusing plane $X . l$ is the distance between the lenticular plane and the detector plane, and $l$ is the distance between the lenticular plane and the refocusing plane. $l^{\prime}=\alpha l$. According to the similarity principle, we extend it to the $4 \mathrm{D}$ light field, the refocusing formula can be expressed

$$
I\left(x^{\prime}, y^{\prime}\right)=\iint L\left(u, v, \frac{1}{\alpha} x^{\prime}+\left(1-\frac{1}{\alpha}\right) u, \frac{1}{\alpha} y^{\prime}+\left(1-\frac{1}{\alpha}\right) v\right) d u d v
$$

If the image originally captured by the camera is not clear, the position of the image plane can be changed by changing the value of the refocusing coefficient, thereby obtaining an image focused at different planes. Figure 2 is a depth-of-field diagram focusing on two different planes.

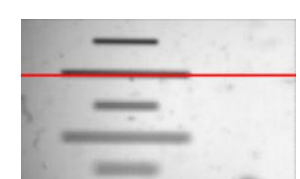

(a)

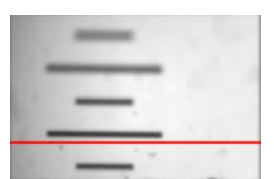

(b)
Figure 2 Images of Refocusing when (a) Focus on Near; (b) Focus on Far

\subsubsection{Depth estimation}

The simplified imaging process of the plenoptic camera 2.0 is shown in Figure 3.

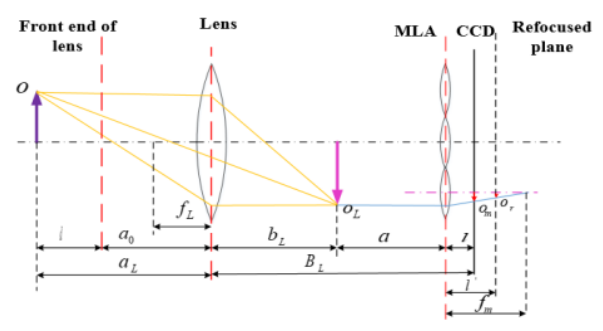

Figure 3 Simplied Imaging Process of the Plenoptic Camera 2.0 
The position $b_{L}$ along the optical axis to which an object at distance $a_{L}$ from the main lens plan is projected, can be calculated by the thin lens equation

$$
\frac{1}{a_{L}}+\frac{1}{b_{L}}=\frac{1}{f_{L}}
$$

Similarly, the position $b$ along the optical axis to which the main lens image at distance $\alpha_{\text {opt }} l$ from the micro lens plane is projected, can be calculated by the thin lens equation

$$
\frac{1}{a}+\frac{1}{\alpha_{o p t} l}=\frac{1}{f_{m}}
$$

Here $\alpha_{\text {opt }}$ indicates a refocusing parameter that make the refocused image of object at the depth $d$ is focused. $l$ is the distance between the microlens plane and the detector plane. $f_{L}$ and $f m$ are the focal length of the main lens and the focal length of the microlens, respectively. The distance from the main plane of the main lens to the imaging detector is $B_{L}, B_{L}$ $=a+b_{L}$. Assuming $a_{L}=a_{0}+d, a_{0}$ is the distance from the front end of the lens, and $d$ is the distance from the object point to the front end of the lens (the depth in this article). Then

$$
d=\left\{f_{L}^{-1}-\left[B_{L}-\left(f_{m}^{-1}-\left(\alpha_{o p t} l\right)^{-1}\right)^{-1}\right]^{-1}\right\}^{-1}-a_{0}
$$

The right side $f_{L}, f m, l$, and $a_{0}$ of the observation equation (5) are fixed parameters of the camera system, which are uniquely determined by the camera and lens used. $d$ is an function of the independent variable $\alpha_{o p t}$ with the remaining parameters are known. So $d$ can be obtained by calculating the camera parameters, or by experiment and curves fitting.

\subsection{D Particle tracking}

\subsubsection{Single-exposure single-frame PTV}

The plenoptic camera uses an appropriate long exposure time to capture the flow field illuminated by the continuous laser, and the resulting image recorded the trajectory of the tracer particles. In order to obtain 3D velocity of the flow field, it is necessary to pre-process all-in-focus image (It is described in detail later). Image preprocessing is mainly divided into five parts from adaptive multi-threshold segmentation to remove crossed trace lines as shown in Figure 4.

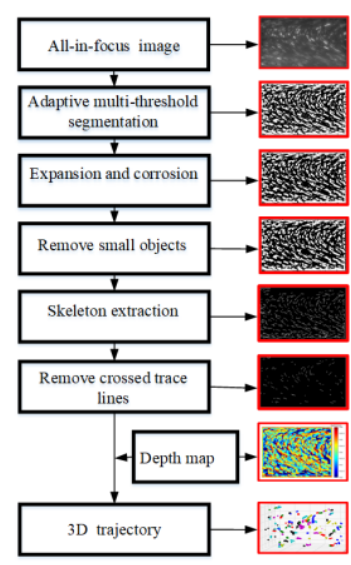

Figure 4 Main Image Preprocessing Flow chart

After obtaining the result map of the image preprocessing, we can get a flow field trace with a width of one pixel, then index the depth of each point in the trace from the depth map, the 3D motion trajectory of the particles can be obtained.

On the obtained trace map of the flow field, the coordinates of all points on each track are known. As shown in figure 6 , the traditional algorithm uses the distance between the start and end points of the trace as the length, and a large error occurs when the track is curved. In this paper, we use the length-finding algorithm similar to the line integral: firstly, all the pixels on the track are arranged in the order of the starting point to the ending point, and then the distance between each two pixels is obtained and finally added to obtain the total track length. It is expressed by

$$
s=\sum_{k} s_{k}
$$

Where $s_{k}$ represents the length of each segment secant $k$ and $s$ represents the total length of each skeleton curve.

For the acquisition of the direction of the speed, the traditional calculation method is to take a straight line connected by the end of the track as a direction vector. However, this method will have a large error when encountering a curved trajectory, and all points on a trajectory share a velocity vector. In this paper, the secant between several pixels before and after the pixel is taken as the velocity direction of the point on the track. The velocity vectors of the individual pixels on the same track are also distinguished, which can well reflect the actual motion of the particles.

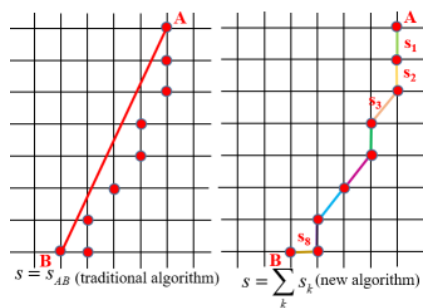

Figure 5 Schematic Diagram of the Difference on Calculating Velocity between Traditional Algorithm and the New

\subsubsection{Double-exposure double-frame PTV}

3D particle locations is required before particle tracking, assuming that the $3 \mathrm{D}$ particle locations for a sequence of images over time have been obtained. In this paper, the relaxation method (Ohmi K et al, 2000) was used to track the located particles in space across frames.

\section{LIGHT FIELD IMAGING SYSTEM AND MEASUREMENT ERROR ANALYSIS}

\subsection{Depth calibration}

The first step in the plenoptic PTV is the calibration of depth. The experimental setup of the calibration system for 
depth estimation using the Raytrix plenoptic camera R12 is shown in Figure 6.

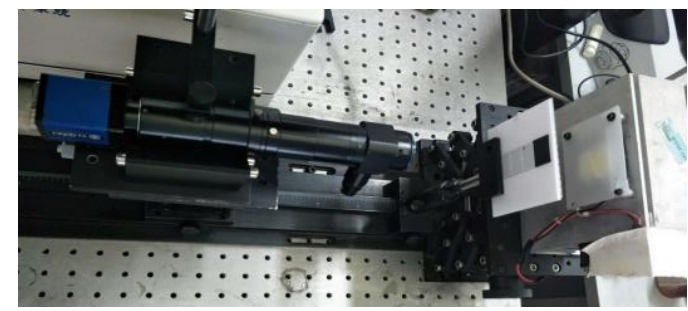

Figure 6 The Photograph of Experimental Setup

The measured object was a calibration plate with round array whose center pitch is $0.32 \mathrm{~mm}$ and diameter is $0.16 \mathrm{~mm}$. The camera is mounted on a one-axis linear translation stage, and the calibration plate is set on a two-axis linear translation stage. The light source was equipped with a piece of diffuser and an active heat sink, it can offer higher quality backlighting. By adjusting the linear translation stages, the raw plenoptic images of calibration plat at different depth plane can be obtained. In order to remove the influence of background noise, five pictures are taken at each depth position, and then summed and averaged as the raw image at the position.

After the multi-distance shooting for the calibration plate carried out by using the system, the captured images are analysed with maximum sharpness principle then the estimated depth corresponding to each image can be obtained.

For each raw image, $\alpha$ is incremented from 0.1 at intervals of $0.0098((5.0-0.1) /(500-1))$ to 5.0,i.e., in the case where the focal plane is always perpendicular to the z-axis(depth-axis), the focal plane position is changed 500 times, so that refocused images at 500 different image planes can be obtained for each raw image. Since all the circles on the calibration plate were in the same depth plane, among all the refocused images, the most sharply focused refocus image is the image corresponding to the ideal imaging plane at the depth position. Correspondingly, the refocusing coefficient $\alpha$ corresponding to the picture with the highest resolution is the optimal refocusing coefficient $\alpha_{\text {opt }}$ corresponding to the working distance.

The standard deviation of the image represents the degree of dispersion of the average gray value of each pixel and the whole image (MAD, 2018). This paper uses the size of the standard deviation of the image to characterize the degree of image clarity.

Measuring the 3D velocity of the flow field requires knowing the 3D particle position. So it is necessary to obtain an all-in-focus image of raw image of the light field. The value of each pixel on the all-in-focus image of the light field camera should be the value of its corresponding object point imaged at the ideal imaging plane (Tao M. W et al, 2013). On the basis of obtaining the depth map, the optimal refocusing coefficient $\alpha_{o p t}$ corresponding to the depth of the point is indexed. Extracting $\alpha_{o p t}$ corresponds to the pixel value of the position in the refocused image, and splicing to obtain a full-focus image. As shown in Figures 7(a), (b) the original partial enlarged view of the light field of the dot calibration plate and the corresponding all-in-focus image .

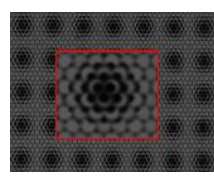

(a)

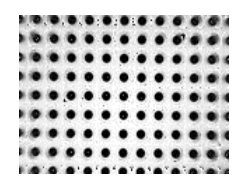

(b)

\section{Figure 7 (a) Raw Image; (b) All-In-Focus Image}

\subsection{Depth calibration results and error analysis}

As shown in Figure 8(a), the corresponding curve of $\alpha$ and sharpness when $d$ is $103 \mathrm{~mm}, 104 \mathrm{~mm}$, and $105 \mathrm{~mm}$ respectively. The corresponding maximum standard deviation $\delta_{\max }$ is $52.10,56.49,56.12$ in order, and $\alpha_{\text {opt }}$ is $2.94,2.48,1.86$. As shown in Figure 8(b), the relationship curve is fitted by least squares method with a fitting coefficient of $0.9939, d$ decreases as $\alpha_{o p t}$ increases. Measuring range is greater than 6 $\mathrm{mm}$. Figure 8(c) shows the error curve with errors less than $5 \%$. Figure $8(d)$ shows the depth resolution curve with a minimum resolution of $0.017 \mathrm{~mm}$ which means that it is possible to distinguish any two objects with a distance greater than $0.017 \mathrm{~mm}$ in the depth direction. It is possible to observe the $3 \mathrm{D}$ tiny structure of the object or the finer phenomenon in the flow field in more detail. The depth resolution at around $103 \mathrm{~mm}$ is significantly higher than others most likely caused by following reason. The reason is that the depth resolution here is obtained from image processing. The resolution at each depth depends on the refocusing factor interval value that can be resolved. When the depth is changed around $103 \mathrm{~mm}$, since the image at the same depth is only refocused 500 times, the refocusing coefficient interval is not sufficiently small, so that the optimal refocusing coefficient $\alpha_{\text {opt }}$ recognized by the image processing program is the same at each depth. Next we consider using Gaussian fitting to improve this situation.

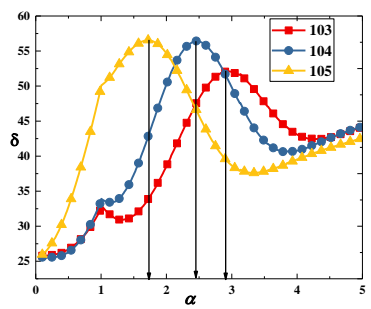

(a)

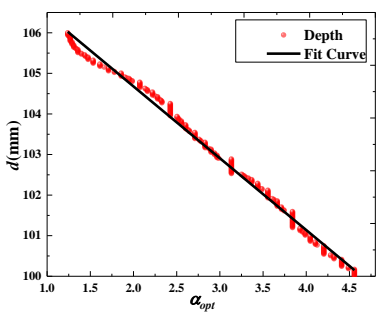

(b)

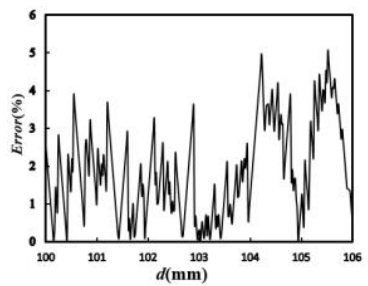

(c) 


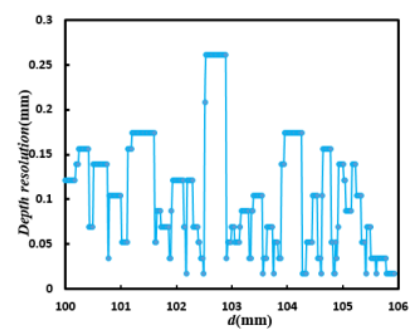

(d)

\section{Figure 8 Analysing Curve of (a) Refocusing Coefficient -Sharpness (b) Depth Calibration (c) Error (d) Depth Resolution}

\subsection{Measurement accuracy analysis of velocity}

To verify the accuracy of the velocity measurement, as shown in Figure 9, a $5 \mu \mathrm{m}$ aperture was placed in front of the point source to simulate the tracer particles in the flow field. A light field camera is used to capture a large number of images of point sources that are driven by the electric rail at a constant speed.

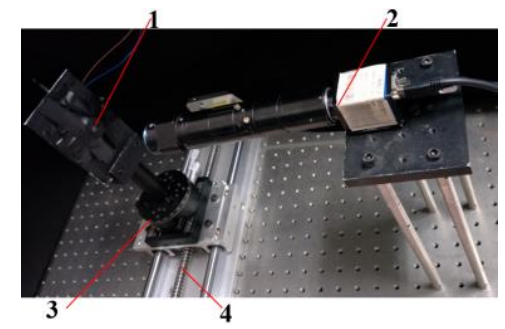

1.Point light source 2. plenoptic camera 3. Angular displacement platform 4.Electric rail

\section{Figure 9 Schematic Diagram of the Experimental System of Point Light}

The velocity of the point source is measured by the method described above, and the variance expression shown in Equation 7 is used to characterize the accuracy of the measurement.

$$
\sigma^{2}=\frac{\sum_{i=1}^{n}\left(v_{i}-\bar{v}\right)}{n}
$$

Where $v_{i}$ is the velocity of each images and $\bar{v}$ is the average velocity. Due to time constraints, in this paper we processed 50 experimental images and $\sigma^{2}=1.4 \times 10^{(-6)}$.

\section{EXPERIMENTS AND RESULTS}

\subsection{Experiment Apparatus}

The schematic diagram of the experimental measurement system is shown in Figure10(a). The experiment is conducted in a low speed circulating water tunnel. The water pump can transport the water with tracking particles from the water tank to the inner barrel of the constant position water tank, and in the experiment the inner barrel is filled and overflowed the water to ensure a constant inlet pressure, that is to ensure the incoming flow of plate is stable. And the inner barrel is connected with the rectangular experimental section, after water flows through the experimental section, the ball valves, the floater flowmeter and back to the water tank, the cycle is formed. Adjusting water flow by the ball valves and observing it by floater flowmeter. The external signal of the signal generator is accepted by plenoptic camera and laser to ensure both of them are synchronized. The belt pulley track drives the camera and laser to move at a constant velocity along the main flow direction of the fluid.

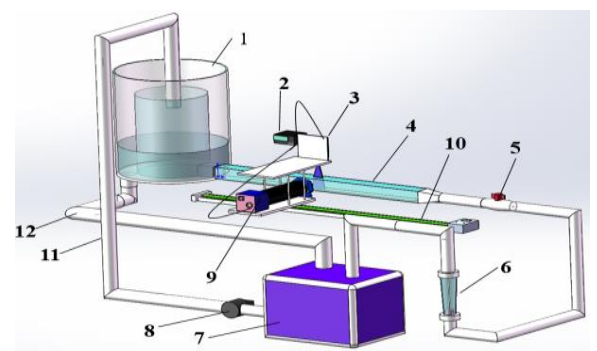

(a)1. Constant position water tank 2. Signal generator 3. Laser 4. Rectangular experiment section 5 the ball valves 6 . floater flowmeter 7 . Water tank 8. water pump 9.plenoptic camera 10. The belt pulley track 11. Inlet pipe 12. Return pipe

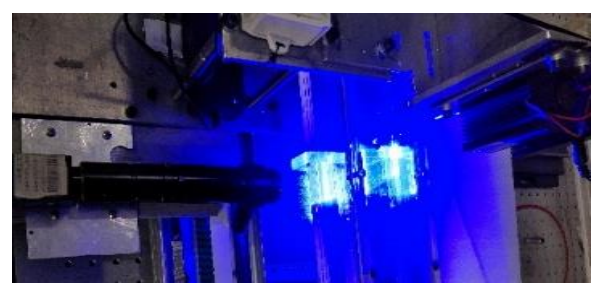

(b)

Figure 10 Schematic Diagram of (a) the Experimental System (b) the Measuring Device

The length of the plexiglass rectangular experimental section is $2500 \mathrm{~mm}$, and the cross section area of the tunnel is $80 \mathrm{~mm} \times 80 \mathrm{~mm}$. A plexiglass plate with the size of $1500 \mathrm{~mm} \times 78 \mathrm{~mm} \times 4.5 \mathrm{~mm}$, is settled in the bottom of the water tunnel. The leading edge of the plate is set to an ellipse with the ratio of the long axis to the short axis as $4: 1$, to alleviate the disturbance of the incoming flow. The tracking particles are polyamide resin (PSP) with an average diameter of $5 \mu \mathrm{m}$, the density of $1.04 \mathrm{~g} / \mathrm{ml}$ and the refractive index of 1.584 . The measurement system is shown in Figure 10(b), consisted of Raytrix R12 plenoptic camera with a pixel size of $3.1 \mu \mathrm{m}$, VSZ-M0108S lens and the laser which is $450 \mathrm{~nm}$ wavelength and $4.8 \mathrm{~W}$. Measuring range is $\mathrm{x}=550 \mathrm{~mm}$ to $\mathrm{x}=1200 \mathrm{~mm}$, here $\mathrm{x}$ is the distance to the front edge of the flat. The speed of incoming flow is about $90 \mathrm{~mm} / \mathrm{s}$ and the speed of camera and laser is set to $88 \mathrm{~mm} / \mathrm{s}$ along the streamwise direction, considering the early study (Qu. 2017).

\subsection{Results and analysis}

In order to solve the high requirements of the laser power of the experiment, the measurement system is moving at a constant velocity to reduce the relative speed of the measurement system and the water flow. When the relative speed is very small, the particle trajectory is basically a point, and the number of pixels occupied will be very few, so that particles with higher gray values are measured under the condition of low power laser, proves that the particles are traced. Figure 11 (a) and (b) are raw plenoptic images captured 
by the plenoptic camera when the camera is still and moving respectively, and the exposure time is $6 \mathrm{~ms}$.

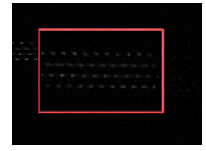

(a)

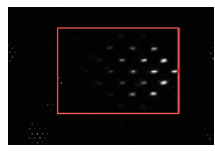

(b)
Figure 11 Raw image of Flow Field When Camera is (a) Still and (b) Moving

In this paper, the single-frame long exposure and doubleframe short exposure methods are used to capture the flow field. Due to the limitations of the implementation conditions, experiments of both methods cannot be performed simultaneously. However, the experimental results of the two methods in the article were carried out at different times under the same test conditions. The whole motion process of the 3D flow field can be obtained by processing the long exposure images. Figure 12(a) and (b) are the depth map and the 3D trajectory map with long exposure. The $\mathrm{x}$ positive direction is the mainstream direction and the $\mathrm{z}$ direction is the depth direction.

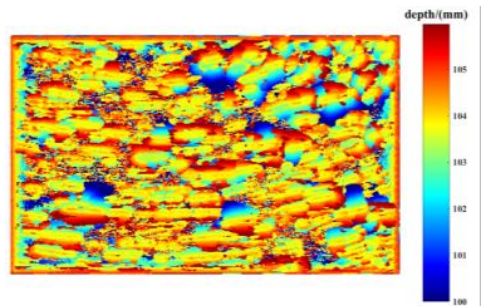

(a)

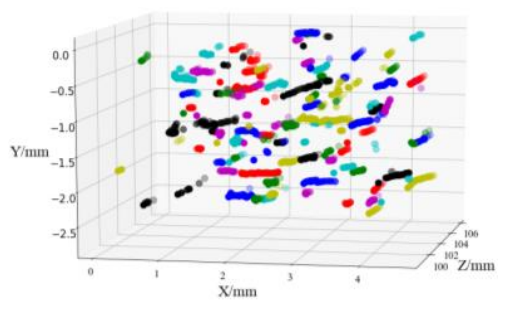

(b)

Figure 12 Single-Frame Single Long Exposure Image of (a) Depth; (b) 3D Trajectory.

3D transient flow field is obtained by processing the short exposure image pairs. The particles in short exposure images can be considered as a feature point. Methods for detecting feature points include Surf (Bay H, 2006), ORB (Rublee E, 2011), FAST (Rublee E, 2006), Harris (Changan KS, 2017), etc. Harris corner detection performs feature point detection by calculating the eigenvalues of the autocorrelation matrix of each pixel. Harris corner detection is partially invariant to image brightness and contrast, and has rotation invariance, but has no scale invariance. Shi-Tomasi (Tatjana Grbić, 2014) improved the Harris corner detection method, directly calculated the eigenvalues of the pixel autocorrelation matrix, and compared the threshold with smaller eigenvalues, and the feature points are larger than the threshold. It is also called the minimum eigenvalue corner detection algorithm. Figure 13(a) shows the 3D particle trajectories found after tracking by using the relaxation method between two different frames without adding the camera's movement speed in the mainstream direction. The purple dots represent the particles in the image of the first frame, and the yellow dots represent the particles in the image of the second frame. These particles are detected by using Shi-Tomasi method. The particles' depth is concentrated between $102 \mathrm{~mm}$ and $105 \mathrm{~mm}$. The thickness of the light sheet is approximate $3 \mathrm{~mm}$, this result confirms the depth algorithm is accurate indirectly. Figure 13(b) and (c) are streamline diagrams of the y direction and the $\mathrm{z}$ direction, respectively, after interpolation of the data obtained after tracking in Figure 13 (a) with adding the camera's movement speed in the mainstream direction. The $\mathbf{u}$ is the velocity of the mainstream direction and the $\mathrm{z}$ direction is the depth direction. The flow direction of the fluid in the depth direction section in Figure 13 (b) is almost the mainstream direction, which is qualitatively consistent with the actual flow field. The flow line in the vertical depth direction of Figure13(c) shows that there may be a vortex, which is qualitatively consistent with the flow field of the 2D camera illuminated by the laser sheet light in the y direction shown in Figure 13(d).

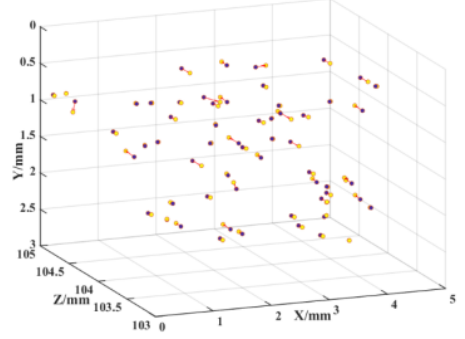

(a)

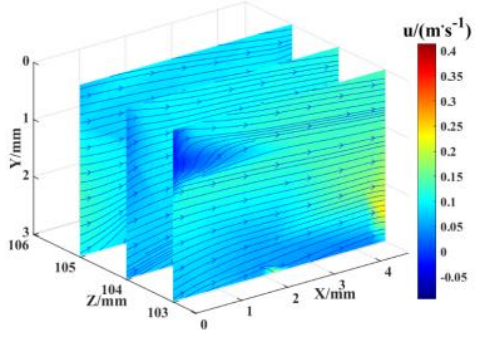

(b)

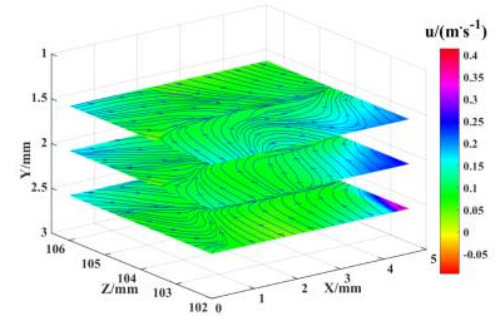

(c)

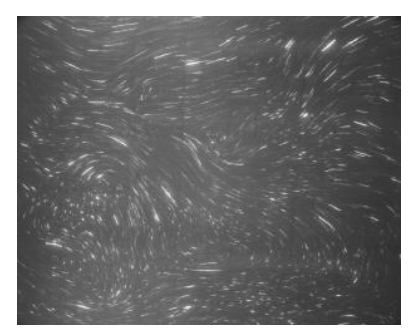

(d) 


\section{Figure 13 Double-Frame Double-Short-Exposure Image of (a) 3D Particles Location and 3D \\ Trajectory; (b) Velocity slices in z direction; (c) Velocity slices in y direction; (d)2D image}

\section{CONCLUSIONS}

The light field 3D PTV technology which offers a simple, single camera solution for volumetric velocity measurements of fluid flow. When it is necessary to track the trajectory of a single particle, especially in the case where the flow field Lagrangian information needs to be obtained and the space is limited, the light field 3D PTV technology shows an advantage. The depth position and the refocusing coefficient are calibrated by taking a dot calibration plate with a known object distance and the center distance of the dot. The depth measurement range is greater than $6 \mathrm{~mm}$ and the error is less than $5 \%$. The minimum depth resolution is $0.017 \mathrm{~mm}$, which means more subtle 3D flow field details can be observed.

The pulley drives the plenoptic camera to follow the fluid movement at a specific speed along the main flow direction of the flow field, which reduces the high frame rate of the camera and the high energy requirement of the laser, thus greatly reduces the experimental cost. Two different PTV methods are used to get both Euler and Lagrange information. The Lagrangian 3D flow field obtained by processing the single long exposure image and 3D transient velocity field obtained by the short exposure combined with the 3D PTV tracking method can be compared and analyzed.

The results show that the plenoptic camera combined with the 3D PTV tracking method can measure the 3D velocity of the turbulent flow well and provide a new method for the study of the flow field mechanism.

\section{REFERENCES}

Bajpayee A. and Techet A. (2013). 3D Particle Tracking Velocimetry (PTV) Using High Speed Light Field Imaging.

Barnard S.T. and Thompson W.B. (1980). Disparity Analysis of Images. IEEE Transactions on Pattern Analysis and Machine Intelligence 2(4):333-340. doi: 10.1109/TPAMI.1980.4767032.

Bay H., Tuytelaars T. and Gool L. V. (2006). SURF: Speeded Up Robust Features - Demonstration. Computer Vision ECCV:404-417. doi: 10.1007/11744023_32.

Changan K. S. and Chilveri P.G. (2017). Stereo image feature matching using Harris corner detection algorithm. 2016 International Conference on Automatic Control and Dynamic Optimization Techniques:691-694. doi: 10.1109/ICACDOT.2016.7877675.

Fahringer T. W., Lynch K. P. and Thurow B. S. (2015). Volumetric particle image velocimetry with a single plenoptic camera. Measurement Science and Technology, 26-115201. doi:10.1088/0957-0233/26/11/115201

Grbić T., Jovanović A., Medić S. and Perović A. (2014). A Note on Feature Extraction Based on Kanade-Shi-Tomasi Procedure and Kalman Filters. 16th International Conference on Speech and Computer. Novi Sad, Serbia:66-73. doi: 10.1007/978-3-319-11581-8_8.
Hoyer K., Holzner M., Lüthi B., Guala M., Liberzon A. and Kinzelbach W. (2005). 3D scanning particle tracking velocimetry. Experiments in Fluids 39(5):923-934. doi: 10.1007/s00348-005-0031-7.

Levoy M. and Hanrahan P. (1996). Light field rendering. Proceedings of ACM SIGGRAPH:31-42. doi: 10.1145/237170.237199.

MA D. (2018). The Research of Image Matching Algorithm Based on Local Feature. Xinjiang University. (in Chinese)

Ng R., Levoy M., Brédif M. and Duval G. (2005). Light field photography with a hand-held plenoptic camera. Stanford University Computer Science Tech Report CSTR 2005-02:1-11. doi: 10.1.1.163.488.

Ohmi K. and Li H. (2000). Particle-tracking velocimetry with new algorithms. Measurement Science and Technology 11(6):603-616.

Ohmi K. Tuladhar S. and Hao J(2016). Light field camera based particle tracking velocimetry[C]//18th International Symposium on the Application of Laser and Imaging Techniques to Fluid Mechanics. July 4-7

Pereira F., Gharib M., Dabiri D. and Modarress D. (2002). Defocusing digital particle image velocimetry: a 3component 3-dimensional DPIV measurement technique. Application to bubbly flows. Experiments in Fluids 29(7):7884. doi: 10.1007/s003480070010.

Pereira F., Stuier H., Graft E.C. and Gharib M. (2006). Two-frame 3D particle tracking. Measurement Science and Technology 17(7):1680-1692. doi: 10.1088/09570233/17/7/006.

Qu N., Cai X. S., Zhou W., Liu C. and Zhou L. (2017). Experimental measurement of coherent structures of turbulent boundary layer by motion single frame imaging method. Journal of Chemical Industry and Engineering 68(11):40884094. doi: 10.11949/j.issn.0438-1157.20170469 (in Chinese)

Rosten E. and Drummond T. Machine Learning for HighSpeed Corner Detection. 9th European Conference on Computer Vision. Graz, AUSTRIA:430-443.

Rublee E., Rabaud V., Konolige K. and Bradski G. (2011). ORB: An efficient alternative to SIFT or SURF. Proceedings of the 2011 IEEE International Conference on Computer Vision:2564-2571. 10.1109/ICCV.2011.6126544.

Satake S. I., Kunugi T., Sato K., Ito T., Kanamori H. and Taniguchi J. (2006). Measurements of 3D flow in a micro-pipe via micro digital holographic particle tracking velocimetry. Measurement Science and Technology 17(7):1647-1651. doi: 10.1088/0957-0233/17/7/002.

Shi S., Ding J., New T. H. and Soria J. J. E. I. F. (2017). Light-field camera-based 3D volumetric particle image velocimetry with dense ray tracing reconstruction technique. 58-78. doi:10.1007/s00348-017-2365-3

Tao M. W., Hadap S., Malik J. and Ramamoorthi R. (2013). Depth from combining defocus and correspondence using light-field cameras. Proceedings of the 2013 IEEE International Conference on Computer Vision. Sydney, Australia:673-680. doi: 10.1109/ICCV.2013.89. 
Willneff J. and Gruen A. (2002). A New SpatioTemporal Matching Algorithm For 3D-Particle Tracking Velocimetry. The 9th of International Symposium on Transport Phenomena and Dynamics of Rotating Machinery. Hawaii,USA, 1-7. 